\title{
Finite Control Set MPC of an AFE Rectifier with Dynamic References
}

\author{
Daniel E. Quevedo, Ricardo P. Aguilera \\ School of Electrical Engineering and Computer Science \\ The University of Newcastle, \\ Callaghan NSW 2308, Australia \\ Email: dquevedo@ieee.org, aguilera.ricardo@gmail.com
}

\author{
Marcelo A. Pérez, Patricio Cortés \\ Departamento de Electrónica \\ Universidad Técnica Federico Santa María, \\ Valparaíso, Chile \\ Email:\{marcelo.perez, patricio.cortes\}@usm.cl
}

\begin{abstract}
We present a model predictive controller for closed loop control of an active front-end rectifier. Our method operates in discrete-time and does not require additional modulators or external control loops. The key novelty of the control algorithm presented lies in the way dynamic references are handled. Simulation studies illustrate that fast and accurate tracking of dynamic dc-voltage and reactive power references can be achieved, while respecting restrictions on maximum power levels of the rectifier.
\end{abstract}

\section{INTRODUCTION}

In many industrial applications active front-end rectifiers (AFEs) have emerged as an attractive topology. When compared to traditional diode-based rectifiers, AFEs allow one to obtain sinusoidal input currents with low harmonic distortion, whilst at the same time providing an accurate rectifier voltage. Moreover, the amount of reactive power drawn from the source can be manipulated in order to reach a unity power factor in the input, or also to compensate a lack of reactive power in the source grid, see [1]. Another area where AFEs play an important role is when a reduction of harmonic distortion is sought. In fact, this converter is widely used as an active filter [2] in which case the AFE is connected in parallel to the nonlinear load thereby generating the harmonic currents necessary to reduce the pollution in the source.

To control AFEs, there exist different methods which are based on pulse-width modulation techniques, both as voltageoriented control (VOC) or as direct power control (DPC), see [3]. The first one works in $d-q$ reference using two control loops. The external loop is based on a Proportional Integral (PI) controller which compensates the dc-voltage error by generating the direct current reference. Afterwards, the dq-current error is compensated in the internal control loop using two PI controllers. These generate dq-voltages which are utilized to produce the associated space vector modulation. The DPC technique also requires two control loops, but in a different manner. In this case, the external controller compensates the dc-voltage error by directly generating the power reference for the internal loop which uses a hysteresis controller. In addition, active and reactive power are estimated using the current measurement. Finally, the switching action to be applied is obtained from a table. The main drawback of both methods is that a linearization of the system around the set point is required in order to adjust the PI controllers.

Recently, in the power electronics area, model predictive control (MPC) strategies have emerged as a promising control technique; see, e.g., [4]. The main advantage of these predictive strategies, when compared to traditional PWM methods, derive from the fact that switching effects can explicitly be taken into account. In fact, so called Finite Control Set MPC formulations, see also [5], use a switching model of the converter to minimize an appropriately chosen cost function. The latter is evaluated at each sampling period by exploring the different switch combinations. The switching action to be applied in the next sampling instant is that which minimizes the cost function used. The cost function can be chosen to reflect various control objectives. Here, one can focus on not only the input currents, but also on other issues including current spectra [6] and number of commutations; see, e.g., [7]. Furthermore, fault detection and reconfiguration ideas can be accommodated, see [8].

In [9] direct power control of an AFE is presented. This method uses a predictive controller in order to obtain the desired active and reactive power in the input. A key issue which arises when controlling an AFE is that dc-voltage and active power levels are coupled. Thus, is is necessary to find compatible references of these variables. For that purpose, in [9], the authors propose to obtain the active power reference from an external PI-controller which is designed to compensate the dc-voltage error. Nevertheless, tuning the external PI controller becomes a difficult task, due to the discrete-time switching nature of the system to be controlled.

The present work presents an MPC formulation for control of AFEs. The key novelty of our method is that it is capable of providing suitable references for the source active power and the rectified voltage, without use of additional control loops. ${ }^{1}$ The proposed method is formulated in discrete-time, uses a state-space of the converter and directly provides the switching action to be applied. As shown via simulations, our formulation allows one to incorporate restrictions on maximum power levels, without incurring any loss of performance due to windup issues, which are typical in PI-loops, see also [11].

\footnotetext{
${ }^{1}$ To some extent, our approach is related to ideas underlying so-called reference governors for model predictive control formulations; see, e.g., [10]
} 
The remainder of this work is organized as follows: In Section II we give a dynamic model of the AFE rectifier. Section III presents the cost function chosen. In Section IV we show how a compatible reference can be formulated. Simulation studies are included in Section V. Section VI draws conclusions.

\section{AFE RECTIFIER}

In the present work, we focus on the AFE rectifier shown in Fig. 1. Here, the rectifier is a three-phase fully-controlled bridge consisting of 6 power transistors connected to a threephase power source $v_{s}$ by means of a filter. The latter is represented by an inductance $L_{s}$ and the parasitic resistance $r_{s}$. The neutral point is electrically floating.

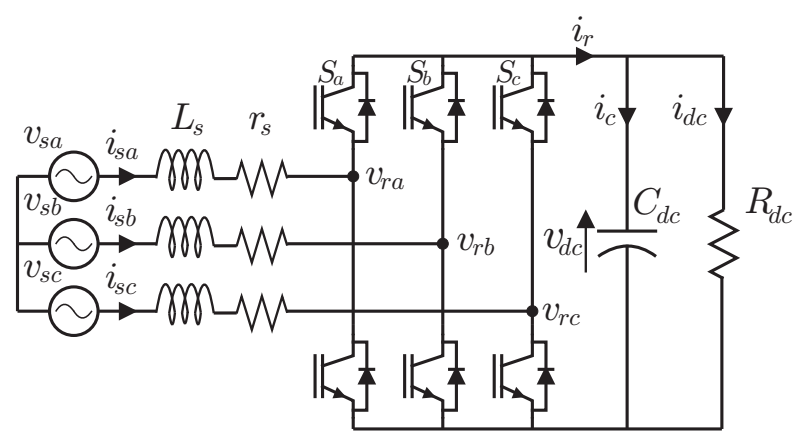

Fig. 1. Active front-end rectifier with floating neutral point

\section{A. Continuous Time Model}

We will adopt an $a b c$-frame and suppose that the three-phase source voltages $v_{s a}, v_{s b}$ and $v_{s c}$ are symmetric, so that in the AFE, we have:

$$
\begin{gathered}
v_{s a}(t)+v_{s b}(t)+v_{s c}(t)=0, \\
i_{s a}(t)+i_{s b}(t)+i_{s c}(t)=0
\end{gathered}
$$

at all times $t \in \mathbb{R}$. Thus, the dynamics of the source current can be represented via:

$$
\begin{aligned}
\frac{d i_{s a}}{d t}= & \frac{1}{L_{s}} v_{s a}(t)-\frac{r_{s}}{L_{s}} i_{s a}(t) \\
& \quad-\frac{1}{3 L_{s}}\left(2 s_{a}(t)-s_{b}(t)-s_{c}(t)\right) v_{d c}(t), \\
\frac{d i_{s b}}{d t}= & \frac{1}{L_{s}} v_{s b}(t)-\frac{r_{s}}{L_{s}} i_{s b}(t) \\
& \quad-\frac{1}{3 L_{s}}\left(-s_{a}(t)+2 s_{b}(t)-s_{c}(t)\right) v_{d c}(t),
\end{aligned}
$$

where the switch variables $s_{a}(t), s_{b}(t)$ and $s_{b}(t)$ are equal to 1 , if at time $t$ the corresponding switch is conducting, and equal to zero, if it is blocking current.
To obtain a dynamic model for the rectified voltage $v_{d c}(t)$, we describe the currents presented on the rectifier side via:

$$
\begin{aligned}
i_{r}(t) & =\left(s_{a}(t)-s_{c}(t)\right) i_{s a}(t)+\left(s_{b}(t)-s_{c}(t)\right) i_{s b}(t), \\
i_{d c}(t) & =\frac{v_{d c}(t)}{R_{d c}} \\
i_{c}(t) & =i_{r}(t)-i_{d c}(t) .
\end{aligned}
$$

Thus, the dynamics of $v_{d c}(t)$ can be modeled via:

$$
\begin{aligned}
\frac{d v_{d c}}{d t}= & \frac{1}{C_{d c}}\left(\left(s_{a}(t)-s_{c}(t)\right) i_{s a}(t)+\left(s_{b}(t)-s_{c}(t)\right) i_{s b}(t)\right) \\
& -\frac{1}{C_{d c} R_{d c}} v_{d c}(t) .
\end{aligned}
$$

\section{B. Discrete Time Model}

The MPC algorithm to be developed operates in discrete time. To obtain a discrete time model of the system, we define the following variable vectors:

$$
\begin{aligned}
\mathbf{i}_{s}(k) & \triangleq\left[\begin{array}{ll}
i_{s a}(k) & i_{s b}(k)
\end{array}\right]^{T}, \\
\mathbf{v}_{s}(k) & \triangleq\left[\begin{array}{ll}
v_{s a}(k) & v_{s b}(k)
\end{array}\right]^{T}, \\
\mathbf{s}(k) & \triangleq\left[\begin{array}{lll}
s_{a}(k) & s_{b}(k) & s_{c}(k)
\end{array}\right]^{T},
\end{aligned}
$$

where $k \in \mathbb{N}$ are the discrete sampling instants.

An Euler approximation of the continuous time model represented by (1) and (2) then gives that:

$$
\begin{aligned}
\mathbf{i}_{s}(k+1) & =\left(1-\frac{r_{s} h}{L_{s}}\right) \mathbf{i}_{s}(k)+\frac{h}{L_{s}}\left(\mathbf{v}_{s}(k)-\mathbf{M s}(k) v_{d c}(k)\right) \\
v_{d c}(k+1) & =\left(1-\frac{h}{C_{d c} R_{d c}}\right) v_{d c}(k)+\frac{h}{C_{d c}}(\mathbf{s}(k))^{T} \mathbf{F} \mathbf{i}_{s}(k),
\end{aligned}
$$

where $h$ is the sampling interval and where

$$
\mathbf{M} \triangleq \frac{1}{3}\left[\begin{array}{ccc}
2 & -1 & -1 \\
-1 & 2 & -1
\end{array}\right], \quad \mathbf{F} \triangleq\left[\begin{array}{cc}
1 & 0 \\
0 & 1 \\
-1 & -1
\end{array}\right]
$$

The model (3) can be written in compact form via:

$$
x(k+1)=A(\mathbf{s}(k)) x(k)+B \mathbf{v}_{s}(k),
$$

where: ${ }^{2}$

$$
\begin{aligned}
A(\mathbf{s}(k)) & \triangleq\left[\begin{array}{cc}
\left(1-\left(r_{s} h\right) / L_{s}\right) I_{2} & -h \mathbf{M} \mathbf{s}(k) / L_{s} \\
\left(h / C_{d c}\right)(\mathbf{s}(k))^{T} \mathbf{F} & 1-h /\left(C_{d c} R_{d c}\right)
\end{array}\right], \\
B & \triangleq\left[\begin{array}{c}
\left(h / L_{s}\right) I_{2} \\
0_{1 \times 2}
\end{array}\right]
\end{aligned}
$$

and where

$$
x(k) \triangleq\left[\begin{array}{c}
\mathbf{i}_{s}(k) \\
v_{d c}(k)
\end{array}\right]
$$

is the state vector of the AFE rectifier.

\footnotetext{
${ }^{2} I_{2}$ denotes the $2 \times 2$ identity matrix, whereas $0_{1 \times 2}=\left[\begin{array}{ll}0 & 0\end{array}\right]$.
} 
It is worth noting that, since each $\mathbf{s}(k)$ only takes values in the finite set

$$
\mathcal{S} \triangleq\left\{\left[\begin{array}{l}
0 \\
0 \\
0
\end{array}\right],\left[\begin{array}{l}
0 \\
0 \\
1
\end{array}\right],\left[\begin{array}{l}
0 \\
1 \\
0
\end{array}\right],\left[\begin{array}{l}
0 \\
1 \\
1
\end{array}\right],\left[\begin{array}{l}
1 \\
0 \\
0
\end{array}\right],\left[\begin{array}{l}
1 \\
0 \\
1
\end{array}\right],\left[\begin{array}{l}
1 \\
1 \\
0
\end{array}\right],\left[\begin{array}{l}
1 \\
1 \\
1
\end{array}\right]\right\},
$$

the system matrix $A(\mathbf{s}(k))$ is also finite set constrained, i.e., we have that $A(\mathbf{s}(k)) \in \mathcal{A}$ for some set $\mathcal{A}$ having eight elements. Discrete-time control design then amounts to choosing the sequences of switch values $\mathbf{s}(k) \in \mathcal{S}, k \in \mathbb{N}$ or, equivalently, the sequence of system matrices $A(\mathbf{s}(k)) \in \mathcal{A}$ for all $k \in \mathbb{N}$.

\section{Model Predictive Control}

In this section, we will present an MPC formulation for the AFE rectifier. To keep things simple, only the discrete-time model (4) will be used. Furthermore, we will choose a cost function which evaluates the effect of the current values $\mathbf{s}(k)$ only. This is computationally attractive and often gives good performance, see, e.g., [4] and references therein. ${ }^{3}$ The cost function chosen quantifies a norm of the tracking error of the three components of the state vector.

From an electrical viewpoint, the main purpose of the AFE is to provide a dc-voltage to the load, whilst at the same time managing a desired balance between active and reactive power. According to the state-space model introduced in Section II-B, for a given system state $x(k)$, the system state, which would result if at time $k$, the switches $s_{a}, s_{b}$ and $s_{c}$ were set to $\mathbf{s}(k) \in \mathcal{S}$ is given by:

$$
x^{\prime}(k+1)=A(\mathbf{s}(k)) x(k)+B \mathbf{v}_{s}(k) .
$$

The associated predicted active power is: ${ }^{4}$

$$
\begin{aligned}
P_{s}^{\prime}(k+1) & =\mathbf{v}_{s}^{T}(k)\left[\begin{array}{ll}
2 & 1 \\
1 & 2
\end{array}\right] \mathbf{i}_{s}^{\prime}(k+1) \\
& =\mathbf{v}_{s}^{T}(k)\left[\begin{array}{lll}
2 & 1 & 0 \\
1 & 2 & 0
\end{array}\right] x^{\prime}(k+1)
\end{aligned}
$$

whereas

$$
\begin{aligned}
Q_{s}^{\prime}(k+1) & =\mathbf{v}_{s}^{T}(k) \sqrt{3}\left[\begin{array}{cc}
0 & 1 \\
-1 & 0
\end{array}\right] \mathbf{i}_{s}^{\prime}(k+1) \\
& =\mathbf{v}_{s}^{T}(k)\left[\begin{array}{ccc}
0 & \sqrt{3} & 0 \\
-\sqrt{3} & 0 & 0
\end{array}\right] x^{\prime}(k+1)
\end{aligned}
$$

denotes the predicted reactive power, and

$$
v_{d c}^{\prime}(k+1)=\left[\begin{array}{lll}
0 & 0 & 1
\end{array}\right] x^{\prime}(k+1)
$$

is the predicted dc-voltage.

The controller proposed in the present work uses the above prediction model to choose the switching values $\mathbf{s}(k)$ which

\footnotetext{
${ }^{3}$ In some situations, the use of a unit switching horizon also gives the optimal solution to a formulation with a larger horizon, see [12].

${ }^{4}$ We approximate $\mathbf{v}_{s}(k+1)=\mathbf{v}_{s}(k)$.
}

minimize a cost function of the form:

$$
\begin{aligned}
J(\mathbf{s}(k))= & \frac{1}{\bar{v}_{d c}^{2}}\left(\tilde{v}_{d c}^{\star}(k+1)-v_{d c}^{\prime}(k+1)\right)^{2} \\
& +\frac{k_{p}}{\bar{P}^{2}}\left(P_{s}^{\star}(k+1)-P_{s}^{\prime}(k+1)\right)^{2} \\
& +\frac{k_{q}}{\bar{P}^{2}}\left(Q_{s}^{\star}(k+1)-Q_{s}^{\prime}(k+1)\right)^{2} .
\end{aligned}
$$

In (5), the superscript ${ }^{\star}$ refers to reference values, $k_{p}$ and $k_{q}$ are tuning parameters, which allow one to trade capacitor voltage reference deviations for deviations in active and reactive power. Finally, $\overline{v_{d c}}$ and $\bar{P}$ are normalizing factors.

In our formulation, we assume that the reference value $Q_{s}^{\star}(k+1)$ and also a reference for the dc-voltage, say $v_{d c}^{\star}(k+1)$, are given. The cost function in (5) uses $Q_{s}^{\star}(k+1)$, but also a reference for the active power, namely $P_{s}^{\star}(k+1)$, and a filtered reference $\tilde{v}_{d c}^{\star}(k+1)$. In the following section, we will show how to obtain $P_{s}^{\star}(k+1)$ and $\tilde{v}_{d c}^{\star}(k+1)$ from $v_{d c}^{\star}(k+1)$ and $Q_{s}^{\star}(k+1)$. The aim is to design references which are consistent and when used in (5) allow the controller to give good performance, despite system constraints.

Remark 1 (Plant State Weighting): It is worth noting that the choice made in (5) amounts to weighting a quadratic form of the tracking error of the predicted state vector. Many theoretical results on MPC algorithms suggest that such a formulation will often lead to closed loops having favorable stability and performance features, see, e.g., [13-17]. How to extend these results to the present case remains an open, and certainly non-trivial, problem.

\section{REFERENCE DESIGN}

As noted in Section I, a key aspect of the situation at hand is that successful tracking of power and voltage references cannot be achieved for arbitrarily chosen and time-varying reference signals. In fact, active power $P_{s}(k)$ and dc-voltage $v_{d c}(k)$ are unavoidably coupled. This relationship is difficult to characterize exactly in closed form, since it is determined not only by electrical parameters of the system, but also by the switching law used.

One way to find compatible reference values has been explored recently in [9], where the use of an additional PI-control loop was examined. It turns out that PI-control gives perfect tracking of constant references in steady state. However, tuning the external controller becomes a difficult task, due to the discrete-time switching nature of the system to be controlled.

In the sequel, we will present a method to provide suitable references for the source power and the rectified voltage. Our formulation uses directly predictive control concepts and does not require an additional control loop. Furthermore, the method proposed allows to incorporate restrictions on maximum power levels, without any integrator windup problems.

Given references $v_{d c}^{\star}(k)$ and $Q_{s}^{\star}(k)$ and the current system state $x(k)$, we seek to find a compatible reference for $P_{s}(k+1)$ and an associated filtered reference value $\tilde{v}_{d c}^{\star}(k+1)$, see Fig. 2. For that purpose, we will examine some electrical properties of 


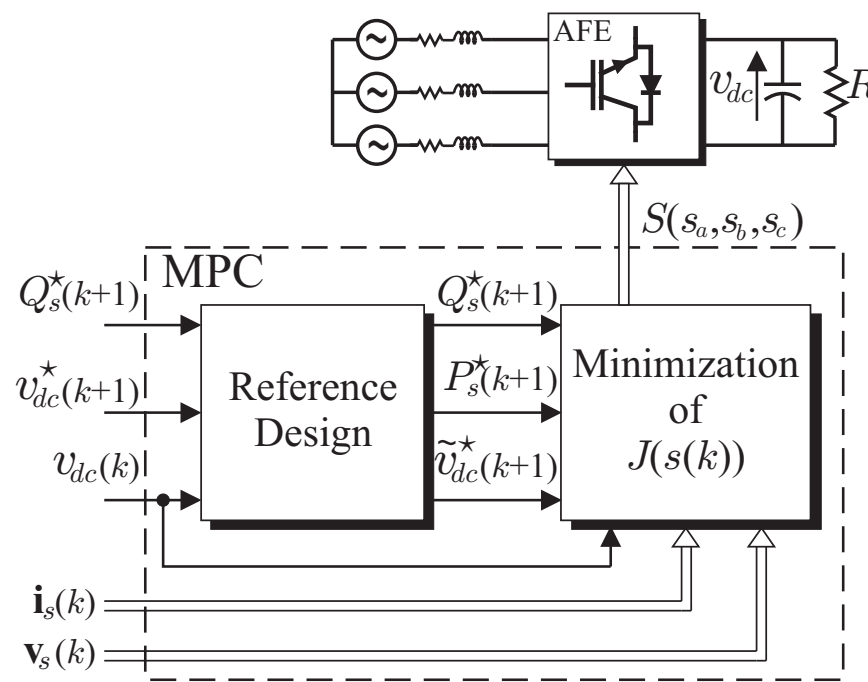

Fig. 2. MPC with Dynamic Reference Design

the AFE topology. We first note that the capacitor voltage $v_{d c}$ can only be adjusted by the capacitor current $i_{c}$. Since the latter quantity cannot be made arbitrarily large, we will introduce a reference prediction horizon, say $N^{\star}$. This horizon value constitutes a design parameter which determines the filtered reference $\tilde{v}_{d c}^{\star}(k+1)$ used in the cost function (5). As shown in Fig. 3, $\tilde{v}_{d c}^{\star}(k+1)$ is obtained by simply allowing the converter to reach $v_{d c}^{\star}(k)$ linearly in $N^{\star}$ steps, i.e., it holds that:

$$
\tilde{v}_{d c}^{\star}(k+1)=v_{d c}(k)+\frac{1}{N^{\star}}\left(v_{d c}^{\star}(k)-v_{d c}(k)\right) .
$$

To find a reference $P_{s}^{\star}$ which is compatible with $\tilde{v}_{d c}^{\star}$, we note that the capacitor current $i_{c}$ needs to ultimately be provided by the AC-source and thereby affects the active input power $P_{s}$. To be more precise, the filtered reference $\tilde{v}_{d c}^{\star}(k+1)$ in (6) requires a capacitor current value of:

$$
i_{c}^{\star}(k+1)=\frac{C_{d c}}{h}\left(\tilde{v}_{d c}^{\star}(k+1)-v_{d c}(k)\right),
$$

which requires a converter current, say $i_{r}^{\star}(k+1)$, given by:

$$
i_{r}^{\star}(k+1)=i_{c}^{\star}(k+1)+\frac{1}{2 R_{d c}}\left(v_{d c}(k)+\tilde{v}_{d c}^{\star}(k+1)\right) .
$$

Therefore, the rectifier power which is necessary to track $\tilde{v}_{d c}^{\star}(k+1)$ satisfies:

$$
\begin{aligned}
P_{r}^{\star}(k+1)= & \tilde{v}_{d c}^{\star}(k+1) i_{r}^{\star}(k+1) \\
= & \left(\frac{1}{2 R_{d c}}+\frac{C}{h}\right)\left(\tilde{v}_{d c}^{\star}(k+1)\right)^{2} \\
& \quad+\left(\frac{1}{2 R_{d c}}-\frac{C}{h}\right) v_{d c}(k+1) \tilde{v}_{d c}^{\star}(k+1) .
\end{aligned}
$$

As noted before, $P_{r}^{\star}(k+1)$ needs to be provided by the AC-source. To obtain the associated value of the active input power reference, namely $P_{s}^{\star}(k+1)$, we will consider only the fundamental component of the source currents. By taking

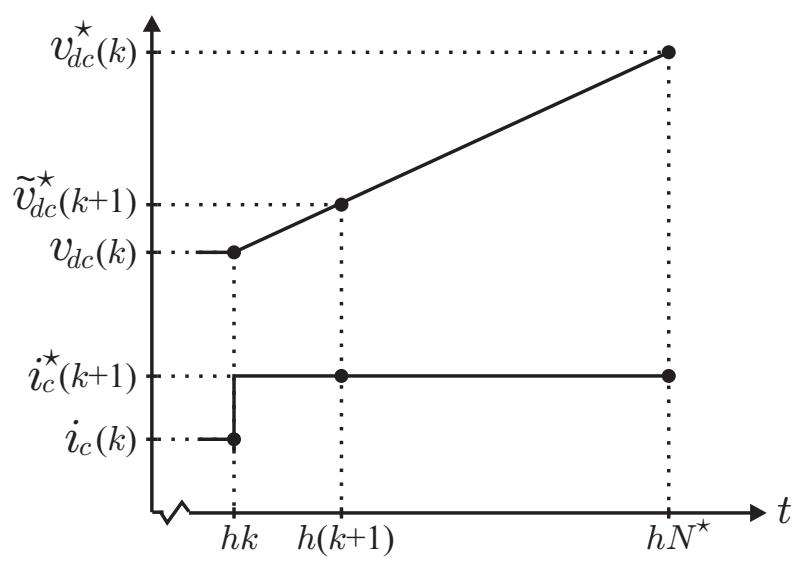

Fig. 3. Dynamic reference design: Capacitor current $i_{c}^{\star}$ needed to increase the capacitor voltage $v_{d c}$ in order to reach the reference $v_{d c}^{\star}$ in $N^{\star}$ time steps.

into account the power losses which occur in the inductor resistances $r_{s}$, we obtain that:

$$
P_{s}^{\star}(k+1)=\frac{2 r_{s}}{3 \hat{V}_{s}^{2}}\left(P_{s}^{\star}(k+1)\right)^{2}+P_{r}^{\star}(k+1),
$$

where $\hat{V}_{s}$ is the source voltage amplitude. The solution to (8) gives the desired reference for $P_{s}(k+1)$, namely:

$$
P_{s}^{\star}(k+1)=\frac{3 \hat{V}_{s}^{2}}{4 r_{s}}\left(1-\sqrt{1-\frac{8 r_{s}}{3 \hat{V}_{s}^{2}} P_{r}^{\star}(k+1)}\right),
$$

where $P_{r}^{\star}(k+1)$ is as in (7).

It is worth emphasizing that, as indicated in (6) and (9), the reference values $P_{s}^{\star}(k+1)$ and $\tilde{v}_{d c}^{\star}(k+1)$ are calculated at each time instant $k$ for a given plant state $x(k)$ and references $v_{d c}^{\star}(k)$ and $Q_{s}^{\star}(k)$. This allows the controller to track dynamic references.

The reference prediction horizon $N^{\star}$ allows the designer to trade-off response times versus control effort. In fact, if a faster tracking response is desired, then $N^{\star}$ should be chosen small. However, this will, in general, lead to large converter currents. To incorporate current limitations, we propose to use the following embellishments of the basic control algorithm:

Remark 2 (Incorporation of current limits): To avoid providing reference values which are associated with large converter currents, one can limit $P_{s}^{\star}(k+1)$ in (9) according to:

$$
\left|P_{s}^{\star}(k+1)\right| \leq P^{\max }(k+1),
$$

where

$$
P^{\max }(k+1) \triangleq \sqrt{\left(3 \hat{V}_{s} \hat{I}_{s}^{\max } / 2\right)^{2}-\left(Q_{s}^{\star}(k+1)\right)^{2}}
$$

and $\hat{I}_{s}^{\max }$ is a limit for the components of $\mathbf{i}_{s}(k)$.

In addition, one can also restrict the possible switch combinations $\mathbf{s}(k)$ when minimizing the cost function $J(\mathbf{s}(k))$ in (5) to those $\mathbf{s}(k) \in \mathcal{S}$, which provide current predictions $\mathbf{i}_{s}^{\prime}(k+1)$ satisfying the limit. The proposed modifications can 
be expected to lead to control loops which are not affected by windup problems typical of PI-control loops, see [18] for discussions on the use of MPC for linear-time invariant systems with convex constraints.

Remark 3: The reference prediction horizon $N^{\star}$ slides forward in time in a moving horizon manner. Viewed from this perspective, the proposed control algorithm can be regarded as having a unit switching horizon, but an overall prediction horizon equal to $N^{\star}$, compare to ideas expressed in [7]. As will be apparent by the simulation studies included in Section $\mathrm{V}$, our formulation gives good performance, but requires only a moderate computational effort.

\section{Simulation Study}

To verify the performance of the predictive controller presented, simulation studies were carried out. The electrical parameters of the AFE rectifier presented in Fig. 1 are chosen as $r_{s}=0.4[\Omega], L_{s}=1[m H], C_{d c}=1000[u F]$ and $R_{d c}=$ $100[\Omega]$. In addition, the source voltage amplitude satisfies $\hat{V}_{s}=\sqrt{2} \cdot 220[\mathrm{~V}]$, whereas the frequency is $f_{o}=50[\mathrm{~Hz}]$. We will consider that the AFE rectifier can tolerate a maximum source current of $\hat{I}_{s}^{\max }=32[A]$.

We use the algorithm proposed in Sections III and IV (including the modification presented in Remark 2) with a sampling period of $h=20[\mu \mathrm{s}]$, and a reference prediction horizon of $N^{\star}=50$. The weighting factors in the cost function $J(\mathbf{s}(k))$ are chosen as $k_{p}=k_{q}=1$.

\section{A. DC-Voltage Tracking}

We first investigate dc-voltage tracking. The performance of the proposed predictive strategy when a change in the dcvoltage reference $v_{d c}^{\star}(k)$ is produced is depicted in Fig 4. Here the system starts with an initial dc-voltage of $v_{d c}=700[\mathrm{~V}]$. To hold this voltage an active source power of the order of $P_{s}=5[k W]$ is required. Moreover, a unity power factor is desired, which results in null reactive power, i.e., $Q^{\star}=0$. As a consequence of this, one can see that the source current $i_{s a}$ is in phase with its corresponding source voltage $v_{s a}$. (In order to appreciate this, the source current has been amplified 10 times in the figure. )

At time instant $t=15[\mathrm{~ms}]$, the dc-voltage reference is changed to $v_{d c}^{\star}=800[v]$. Since the reference prediction horizon is $N^{\star}=50$ and the sampling period is $h=20[\mu \mathrm{s}]$, one could expect that the new reference be reached in about $1 \mathrm{~ms}$. However, since the source current is limited to $32[A]$, this is not possible. With the proposed control formulation, source current limits are incorporated into the reference design as power reference limits, which in this case are give by $P^{\max }=15[k W]$, see (10). It can be seen in Fig. 4 that the system takes about $10 \mathrm{~ms}$ to reach the new dc-voltage reference. As can be appreciated, the proposed controller gives good tracking performance, with no overshoot. Throughout the transient where saturations occur, the reactive power $Q_{s}$ has been held equal to zero by the controller, keeping the source current in phase with its associated voltage.
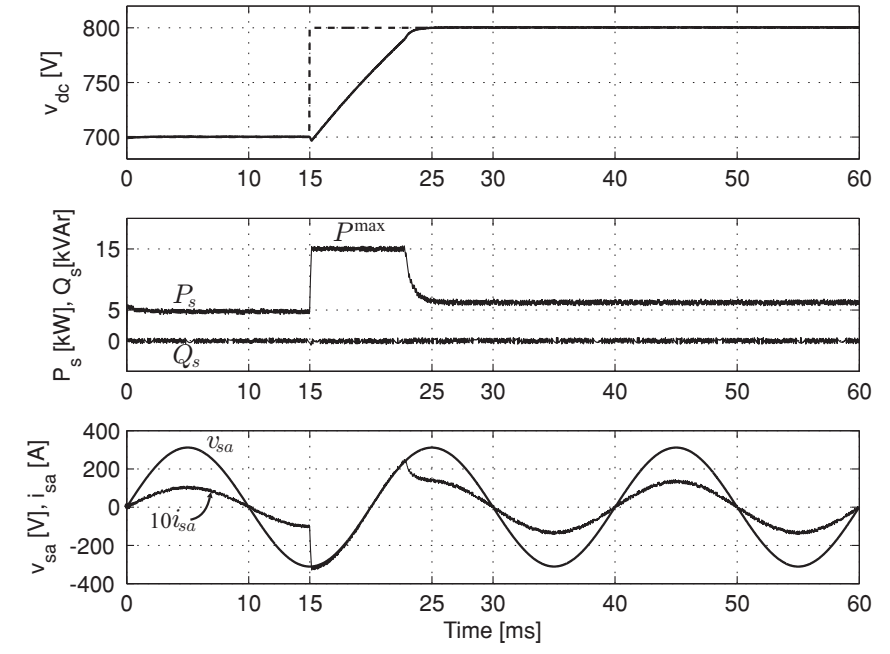

Fig. 4. Performance of the MPC algorithm presented: step in the (unfiltered) dc-voltage reference $v_{d c}^{\star}$.

\section{B. Tracking of Dynamic Reactive Power References}

Since AFE converters are also used to compensate for the lack of reactive power that the electrical grid can present, it is important to verify that the proposed controller can maintain the rectified voltage $v_{d c}(t)$ even when different power factors are required. Fig. 5 depicts the performance of our proposal facing this situation. Similar to the previous test, the initial condition of the system considers a dc-voltage of $v_{d c}=700[\mathrm{~V}]$ and an active power in the order of $P_{s}=5[\mathrm{~kW}]$. However, a reactive power of $Q_{s}=-2.5[\mathrm{kVAr}]$ is provided from the rectifier to the source, amounting to a negative power factor of $\mathrm{PF}=-0.89$. Consequently, the source current leads the source voltage by about $27^{\circ}$.

Next, at the instant $t=25[\mathrm{~ms}]$, a step up change in the reactive power reference is produced, namely from $Q_{s}=$ $-2.5[\mathrm{kVAr}]$ to $Q_{s}=2.5[\mathrm{kVAr}]$. Here one can see that the controller makes the system track this step change quickly, barely affecting the active power $P_{s}$ or the capacitor voltage $v_{d c}$. The power factor now is positive $(\mathrm{PF}=0.89)$ and the source current now lags the source voltage with the same angle of $27^{\circ}$.

\section{Load Changes}

Another important aspect to be analyzed is the compensation of load changes. Simulation results are depicted in Fig. 6. Here we have the same initial condition as in the first case, namely unity power factor, $Q_{s}=0[\mathrm{kVAr}]$, and a rectified voltage of $v_{d c}=700[\mathrm{~V}]$, requiring an active power of about $P_{s}=5[k W]$.

At the instant $t=25[\mathrm{~ms}]$, a resistance load change from $R_{d c}=100[\Omega]$ to $R_{d c}=50[\Omega]$ is produced. This generates a slight dc-voltage drop which is compensated, as expected, in about $1 \mathrm{~ms}$. (In this case, power levels needed are safely within the limits of the AFE.) 

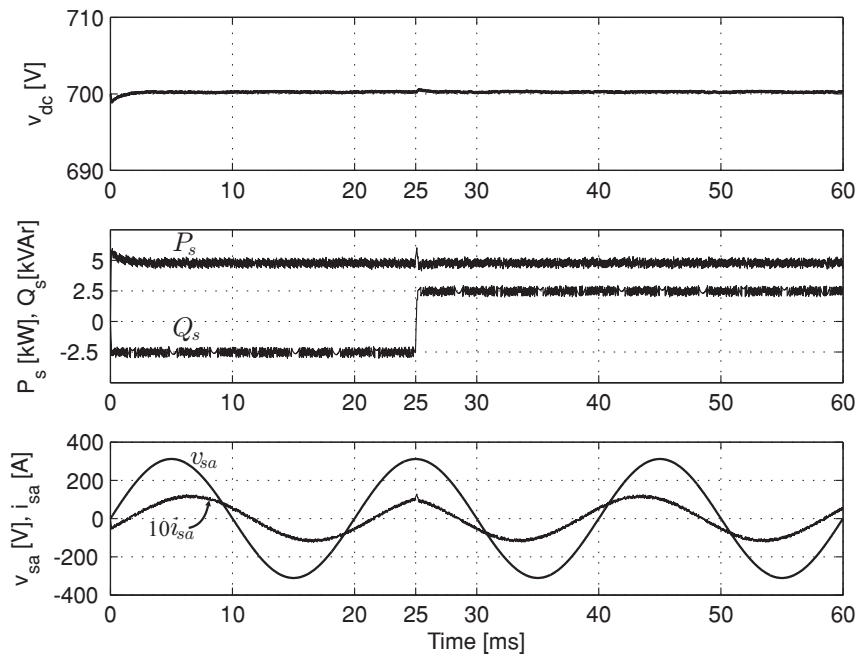

Fig. 5. Performance of the MPC algorithm presented: step in the reactive power reference $Q_{s}^{\star}$
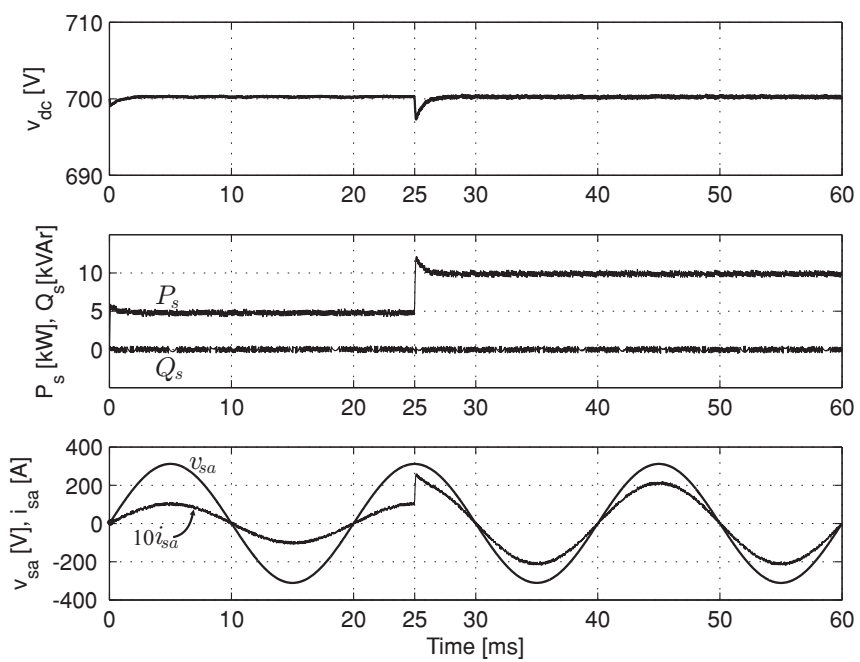

Fig. 6. Performance of the MPC algorithm presented: step in the load resitance $R_{d c}$.

\section{CONCLUSiOnS}

We have presented a Model Predictive Control formulation for AFE rectifiers. The proposed control algorithm operates in discrete-time and does not require any additional modulators to drive the switches. The switching horizon is chosen equal to one. Thus, the search set for on-line optimizations has only eight elements, making the switching signals easy to calculate.

The key novelty of our approach has been to incorporate dynamic reference handling directly. More precisely, careful examination of electrical properties of the rectifier topology has allowed us to elucidate the issue of finding compatible references for active source power and dc-voltage.

The control architecture proposed requires no external control loops and also incorporates possible saturations of source currents directly in its formulation. Here the introduction of a reference prediction horizon, which may differ from the switching horizon, has proven useful, allowing the system designer to trade-off tracking bandwidth for control effort.

Simulation results indicate that tracking of dynamic dcvoltage and power references and also compensating of load changes is possible. In fact, good transient performance can be achieved even when saturation limits are reached.

\section{ACKNOWLEDGMENTS}

Marcelo Pérez and Patricio Cortés acknowledge the support of the Chilean Research Council under grant Fondecyt 11090253 and of the Universidad Técnica Federico Santa María.

\section{REFERENCES}

[1] J. R. Rodríguez, J. W. Dixon, J. R. Esponoza, J. Pontt, and P. Lezana, "PWM regenerative rectifiers: state of the art," IEEE Trans. Ind. Electron., vol. 52, no. 1, pp. 5-22, Feb. 2005.

[2] S. Kwak and H. A. Toliyat, "Design and rating comparisons of PWM voltage source rectifiers and active power filters for AC drives with unity power factor," IEEE Trans. Power Electron., vol. 20, no. 5, pp. 1133-1142, Sept. 2005.

[3] M. Malinowski, M. P. Kazmierowski, and A. M. Trzynadlowski, "A comparative study of control techniques for PWM rectifiers in AC adjustable speed drives," IEEE Trans. Power Electron., vol. 18, no. 6, pp. 1390-1396, Nov. 2003.

[4] P. Cortés, M. P. Kazmierowski, R. M. Kennel, D. E. Quevedo, and J. Rodríguez, "Predictive control in power electronics and drives," IEEE Trans. Ind. Electron., vol. 55, no. 12, pp. 4312-4324, Dec. 2008.

[5] S. Kouro, P. Cortés, R. Vargas, U. Amman, and J. R. Rodríguez, "Model predictive control: A simple and powerful method to control power converters," IEEE Trans. Ind. Electron., vol. 56, no. 6, pp. 1826-1838, June 2009.

[6] P. Cortés, J. Rodríguez, D. E. Quevedo, and C. Silva, "Predictive current control strategy with imposed load current spectrum," IEEE Trans. Power Electron., vol. 23, no. 2, pp. 612-618, Mar. 2008.

[7] T. Geyer, G. Papafotiou, and M. Morari:, "Model predictive direct torque control-part I: Concept, algorithm and analysis," IEEE Trans. Ind. Electron., vol. 56, no. 6, pp. 1894-1905, June 2009

[8] R. P. Aguilera, D. E. Quevedo, T. J. Summers, and P. Lezana, "Predictive control algorithm robustness for achieving fault tolerance in multicell converters," in Industrial Electronics, 2008. IECON 2008. 34th Annual Conference of IEEE, Nov. 2008, pp. 3302-3308.

[9] P. Cortés, J. Rodríguez, P. Antoniewicz, and M. Kazmierkowski, "Direct power control of an AFE using predictive control," IEEE Trans. Power Electron., vol. 23, no. 5, pp. 2516-2522, Sept. 2008.

[10] A. Bemporad, "Reference governor for constrained nonlinear systems," IEEE Trans. Automat. Contr., vol. 43, no. 3, pp. 415-419, 1998.

[11] K. J. Åström and T. Hägglund, PID Controllers: Theory, Design and Tuning, 2nd ed. Research Triangle Park, N.C.: Instrument Society of America, 1995.

[12] D. E. Quevedo, C. Müller, and G. C. Goodwin, "Conditions for optimality of naïve quantized finite horizon control," Int. J. Contr., vol. 80, no. 5, pp. 706-720, May 2007.

[13] D. Q. Mayne, J. B. Rawlings, C. V. Rao, and P. O. M. Scokaert, "Constrained model predictive control: Optimality and stability," Automatica, vol. 36, no. 6, pp. 789-814, June 2000.

[14] J. M. Maciejowski, Predictive Control with Constraints. Englewood Cliffs, N. J.: Prentice-Hall, 2002.

[15] E. F. Camacho and C. Bordons, Model Predictive Control. New York, N.Y.: Springer-Verlag, 1999.

[16] G. C. Goodwin, M. M. Serón, and J. A. De Doná, Constrained Control \& Estimation - An Optimization Perspective. London: Springer Verlag, 2005.

[17] D. E. Quevedo, G. C. Goodwin, and J. A. De Doná, "Finite constraint set receding horizon quadratic control," Int. J. Robust Nonlin. Contr. vol. 14, no. 4, pp. 355-377, Mar. 2004.

[18] J. A. De Doná, G. C. Goodwin, and M. M. Serón, "Anti-windup and model predictive control: Reflections and connections," European J. Contr., vol. 6, no. 5, pp. 467-477, 2000 